\title{
Resonant energy transfer between two defects in a photonic-band-gap system
}

\author{
Yui Chow, Lie-Ming Li, ${ }^{*}$ Zhao-Qing Zhang, and C. T. Chan \\ Department of Physics, Hong Kong University of Science and Technology, Clear Water Bay, Hong Kong, China
}

(Received 2 November 1998; revised manuscript received 1 March 1999)

\begin{abstract}
The wave interference behaviors between two interacting defects in a two-dimensional photonic-band-gap system are studied by using the multiple-scattering method. By injecting electromagnetic wave energy at one defect, we find oscillatory resonant energy transfer between two defects when both defect mode frequencies lie inside a full gap. When one of the defect mode frequency merges with the pass band forming a resonant state, energy leakage as well as frequency modulation are observed due to the interaction among the defect mode, the resonant states, and the band states. These classical resonant behaviors are realizable in simple twodimensional photonic-band-gap systems. [S0163-1829(99)02835-0]
\end{abstract}

\section{INTRODUCTION}

Resonant energy transfer between two (or more) localized quantum states through potential barrier(s) is not uncommon in nature. The umbrella mode of an ammonia molecule is a famous example. ${ }^{1}$ In glasses and certain amorphous materials, the model of a two-level system has been adopted to explain the linear temperature dependence of specific heat at low temperature. ${ }^{2}$ Although the resonant energy transfer is a natural consequence of elementary quantum mechanics, a direct experimental observation of these phenomena is difficult for atomic systems, and are frequently complicated by manybody interactions. Here we propose a simple system to realize the classical analog of quantum resonant phenomena. Our system is a two-dimensional (2D) photonic-band-gap (PBG) material with two interacting defects. PBG materials have been under intensive studies both theoretically and experimentally over the past few years. ${ }^{3-13}$ The existence of photonic gaps, which prohibit the propagation of electromagnetic (EM) waves, can have important impacts both in science and technology. On the fundamental side, the presence of a spectral gap facilitates the localization of EM waves when randomness is added to the system..$^{5}$ The absence of propagating modes inside a gap can also produce distinctive quantum electrodynamic effects. ${ }^{14-17}$ For instance, the spontaneous emission of an excited atom will be suppressed if the transition frequency lies inside such a gap. When the transition frequency is near a band edge, it has also been predicted that strong coupling between the atom and photons can lead to anomalous Lamb shift of the excited level and the splitting of the level into a doublet. When one level lies inside the gap and the other lies outside the gap, interesting resonance fluorescence phenomenon has been predicted. ${ }^{15}$ There may exist a fractional steady-state population of excited states even when the transition frequency lies outside the gap. Such interesting resonant behaviors have also been studied for a model of three-level atoms. ${ }^{16,17}$

\section{PHYSICAL SYSTEM AND METHOD OF CALCULATION}

Let us consider a 2D PBG system with two interacting defects. We demonstrate here that the resonance between these two defects is capable of producing similar behaviors found in quantum-mechanical systems. The transmission properties of 2D PBG systems with two or more defects have been studied previously. ${ }^{8-10}$ In the case when the separation between two defects is larger than the linear size of the single defect mode, two defects are uncoupled and their defect frequencies become degenerate. When two defects are close enough, the interaction between two defects splits the degeneracy and produces two different defect frequencies. In the study of resonant energy transfer, we consider two interacting defects. We inject a pulse from one of the defects and study the time dependence of the total energy stored in each defect. Multiple-scattering theory is used to calculate the response function of the electric field in the frequency domain. Its time dependence is obtained by taking the Fourier transform.

Consider a system of $N$ identical cylinders (assumed to be infinite along the axis) of radius $R$ and dielectric constant $\epsilon$ under an external source $u_{\text {inc }}(\boldsymbol{\rho})$ at a fixed frequency $\omega$ $=2 \pi f$. Here $\boldsymbol{\rho} \equiv(\rho, \theta)$ specifies the position of a point in a $2 \mathrm{D}$ plane. We consider here the case of the TM mode and $u$ denotes the electric field polarized along the cylindrical axis. The details of the multiple-scattering theory are given elsewhere. ${ }^{13}$ Here, we summarize the main formulas. In the presence of an external field, the total field, which is the sum of external field $u_{\text {inc }}(\boldsymbol{\rho})$ and radiation from all cylinders, can be written in the form

$$
\begin{aligned}
u(\rho)= & \sum_{j=1}^{N} \sum_{m=-M}^{M} \alpha_{m}(j)\left[J_{m}\left(k_{0} \rho_{\rho j}\right)\right. \\
& \left.+D_{m}(j) H_{m}\left(k_{0} \rho_{\rho j}\right)\right] e^{i m \theta_{\rho j}}
\end{aligned}
$$

where $k_{0}=\omega / c, \boldsymbol{\rho}_{\rho j}=\boldsymbol{\rho}-\boldsymbol{\rho}_{j}=\left(\rho_{\rho j}, \theta_{\rho j}\right)$, and $\boldsymbol{\rho}_{j}$ represents the center of the $j$ th cylinder. $J_{m}$ and $H_{m}$ are the Bessel and the Hankel function of the first kind and $D_{m}(j)$ has the form $^{16}$

$$
D_{m}(j)=\frac{k J_{m}\left(k_{0} R\right) J_{m}^{\prime}(k R)-k_{0} J_{m}^{\prime}\left(k_{0} R\right) J_{m}(k R)}{k_{0} J_{m}(k R) H_{m}^{\prime}\left(k_{0} R\right)-k H_{m}\left(k_{0} R\right) J_{m}^{\prime}(k R)} .
$$

The function $\alpha_{m}(j)$ is the solution of the following selfconsistent equations: 


$$
\begin{aligned}
\alpha_{m}(j)= & \alpha_{m}^{(0)}(j) \\
& +\sum_{i \neq j} \sum_{l=-M}^{M} \alpha_{l}(i) D_{m}(i) e^{i(l-m)\left(\theta_{j i}+\pi\right)} H_{l-m}\left(k_{0} \rho_{j i}\right),
\end{aligned}
$$

where $\boldsymbol{\rho}_{j i}=\boldsymbol{\rho}_{i}-\boldsymbol{\rho}_{j}=\left(\rho_{j i}, \theta_{j i}\right)$, and the function $\alpha_{m}^{(0)}(j)$ represents the expansions of the external field $u_{\text {inc }}(\boldsymbol{\rho})$ about the center of the $j$ th cylinder, i.e.,

$$
u_{\text {inc }}(\boldsymbol{\rho})=\sum_{m=-M}^{M}\left[\alpha_{m}^{(0)}(j) J_{m}\left(k_{0} \rho_{\rho j}\right)\right] e^{i m \theta_{\rho j}} .
$$

If we rewrite Eq. (1) as $u=u_{\text {inc }}+u_{\text {scatt }}$, then the total scattered field becomes

$$
u_{\text {scatt }}(\boldsymbol{\rho})=\sum_{j=1}^{N} \sum_{m=-M}^{M} \alpha_{m}(j) D_{m}(j) H_{m}\left(k_{0} \rho_{\rho j}\right) e^{i m \theta_{\rho j}}
$$

The radiation pattern can be obtained from the Poynting vector

$$
\mathbf{S}(\boldsymbol{\rho})=\frac{c}{8 \pi k_{0}} \operatorname{Im}\left[u(\boldsymbol{\rho}) \nabla u^{*}(\boldsymbol{\rho})\right] .
$$

In far field, when $k_{0} \rho \gg 1$, the asymptotic behavior of $u_{\text {scatt }}$ in Eq. (1) becomes $u_{\text {scatt }}(\boldsymbol{\rho}) \rightarrow f_{s}(\theta) \exp \left(i k_{0} \rho\right) / \sqrt{\rho}$, where $f_{s}(\theta)$ is the total scattering amplitude and has the form

$$
\begin{aligned}
f_{s}(\theta)= & \sqrt{\frac{2}{\pi k_{0}}} e^{-i \pi / 4} \sum_{i=1}^{N} \sum_{m=-M}^{M} \\
& \times e^{-i k_{0} \rho_{i} \cos \left(\theta_{i}-\theta\right)}(-i)^{m} e^{i m \theta} \alpha_{m}(i) D_{m}(i) .
\end{aligned}
$$

We consider a circular PBG sample of radius $R_{s}$. Inside this circular domain, cylinders of radius $R$ and dielectric constant $\epsilon$ are arranged in a square array with lattice constant $a$. In this work we have chosen $R_{s}=6 a, \epsilon=10$, and $R / a$ $=0.309$, which gives a filling fraction $x=0.3$. The geometry of the sample is shown in Fig. 1(a). We introduce defect modes by replacing two nearest-neighbor cylinders at the center of the sample by two defect cylinders with a different dielectric constant $\epsilon_{d}$ [marked by two dark circles in Fig. 1(a)]. The wave energy is injected into the defect sitting at the center of the sample. For a continuous wave source centered at the origin, the incident wave for all other cylinders is a result of radiation from the centered cylinder, which can be expressed in the form

$$
u_{\mathrm{inc}}(\boldsymbol{\rho})=\sum_{m=-\infty}^{\infty} A_{m} H_{m}\left(k_{0} \rho\right) e^{i m \theta}
$$

In far field, we have $u_{\text {inc }}(\boldsymbol{\rho}) \sim a_{0}(\theta) \exp \left(i k_{0} \rho\right) / \sqrt{\rho}$, where

$$
a_{0}(\theta)=\sqrt{\frac{2}{\pi k_{0}}} \sum_{m=-\infty}^{\infty} A_{m} e^{-i \pi / 4}(-i)^{m} e^{i m \theta} .
$$

From Eq. (6), the far-field energy flux has the form

$$
\mathbf{S}(\rho, \theta)=\frac{c\left[\left|a_{0}(\theta)+f_{s}(\theta)\right|\right]^{2}}{8 \pi \rho} \hat{\rho}=S(\theta) \frac{\hat{\rho}}{\rho} .
$$
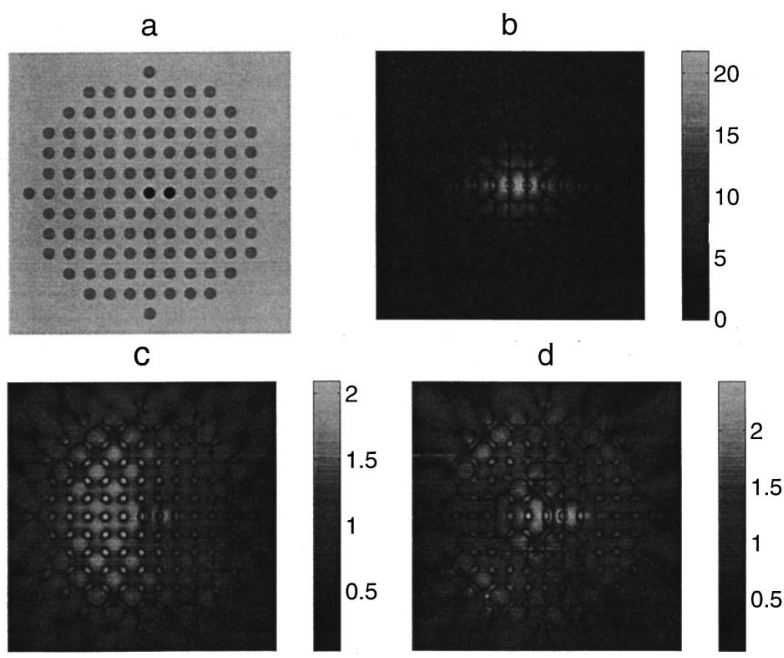

FIG. 1. (a) The sample geometry consists of cylinders in a square lattice. The dark circles denote the positions of defects. The amplitude maps for the cases of defect dielectric constant $\epsilon_{d}=4.4$ are shown in (b), (c), and (d) for the defect state $(\hat{f}=0.498)$, bandedge state $(\hat{f}=0.544)$, and the resonant state $(\hat{f}=0.556)$.

From Eq. (10), we can calculate the total radiation power $P=\int_{0}^{2 \pi} S(\theta) d \theta$. The spectrum of $P$ determines the full gap as well as the defect frequencies of the system. For frequencies inside a full gap, the density of states is zero, which in turn gives a divergent input impedance and vanishing $P$. Therefore, a gap in the spectrum of $P$ represents the full gap of the system. On the other hand, at defect frequencies, the presence of sharply localized modes at the injection point gives rise to a small input impedance and, therefore, produces a large peak in the radiation power. Thus, from its peak positions of $P$ we can identify the defect frequencies.

\section{RESULTS AND DISCUSSIONS}

For simplicity, we consider only the case of a line source which produces EM waves with amplitude $u_{\text {inc }}(\boldsymbol{\rho})$ $=H_{0}\left(k_{0} \rho\right)$. This line source can be produced from the dipole oscillation in the direction parallel to the cylinder axis. In order to examine the validity of this approach, we first calculated $P$ when the sample is defect free. From the calculated $P$, we found a gap between 0.431 to 0.542 in the dimensionless units of $\hat{f} \equiv \omega a / 2 \pi c$. This is the second gap of the system. The location of this gap agrees perfectly well with the results of density-of-states calculations. In curve $A$ of Fig. 2, we plot the logarithm of $P$ for the case of two nearest-neighbor defects with $\epsilon_{d}=6$. Two sharp defect modes appear inside the gap at defect frequencies $\hat{f}=0.459$ and 0.513 . The dependence of these defect frequencies on $\epsilon_{d}$ is shown by two dashed lines in Fig. 3 from $\epsilon=4.0$ to 7.0. The two solid lines in Fig. 3 represent the lower and upper band edges of the gap. The defect frequency increases as $\epsilon_{d}$ decreases. However, when $\epsilon_{d} \leqslant 5$, the upper defect state moves into the band and forms a resonant state. In curves $B$ and $C$ of Fig. 2, we also plot the logarithm of $P$ for the cases of $\epsilon_{d}=5.0$ and 4.4, respectively. For curve $B$, there is only one defect state at $\hat{f}=0.482$. The other defect state has merged with the state at the band edge and produces a large 


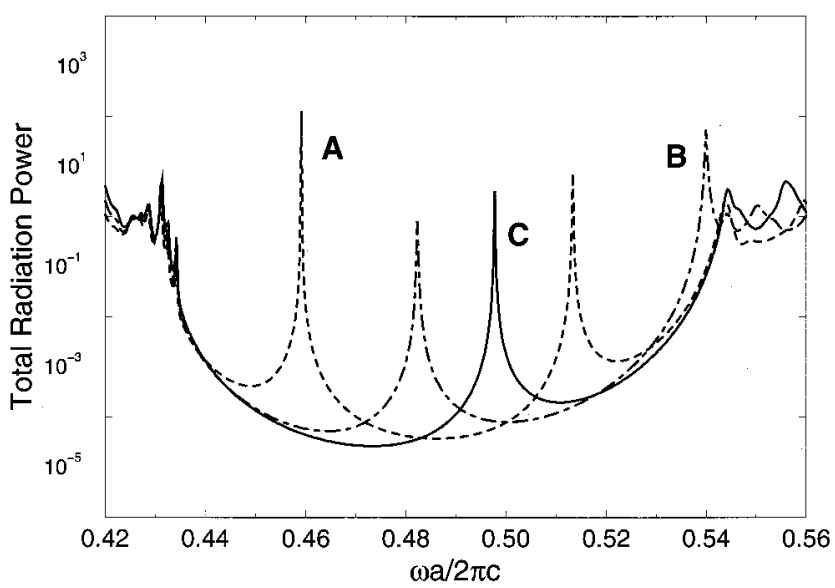

FIG. 2. The logarithm of the total radiation power for the systems discussed in the text. Curves $A, B$, and $C$ are the results with two defects of the dielectric constant $\epsilon_{d}=6.0,5.0$, and 4.4, respectively.

peak at $\hat{f}=0.540$. For curve $C$, the lower defect frequency is increased to $\hat{f}=0.498$, and the upper one has moved into the band and formed a resonant state at $\hat{f}=0.556$. The peak at $\hat{f}=0.544$ represents states at band edge. In order to see the nature of states at these three different peak positions, we plot in Figs. 1(b)-1(d) the mode-amplitude maps $|u(\boldsymbol{\rho})|^{2}$ for the case of $\epsilon_{d}=4.4$ at $\hat{f}=0.498,0.544$, and 0.556 , respectively. In Fig. 1(b), a mode localized around defect cylinders is clearly seen for the defect state. Figure 1(d) shows a state with a large amplitude near the defects superimposed on a rather uniform background across the sample, which is quite typical for a resonant state. For the state at the band edge [Fig. 1(c)], the mode shows a pass band character with small amplitude contrast between the defect and the normal cylinders. The asymmetry in the amplitude map is due to the asymmetric arrangement of energy injection of the two defect cylinders.

In order to study the energy transfer between two defect cylinders, instead of using cw waves, we inject a Gaussian pulse which has a spectrum centered at the frequency $\hat{f}_{c}$ and

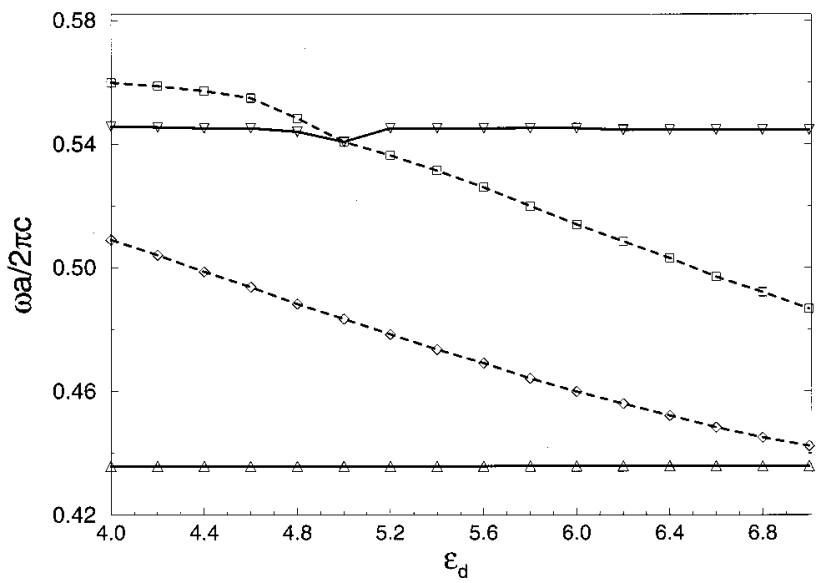

FIG. 3. As a function of defect dielectric constant $\epsilon_{d}$, two dashed lines represent the defect frequencies and two solid lines represent the states at the band edge. a width $\hat{\delta}$. The space-time dependence of the field response function can be expressed as

$$
\widetilde{u}(\boldsymbol{\rho}, \hat{t})=\frac{1}{\sqrt{2 \pi} \hat{\delta}} \int u(\boldsymbol{\rho}, \hat{f}) e^{-\left(\hat{f}-\hat{f}_{c}\right)^{2} / 2 \hat{\delta}^{2}} e^{-i \hat{f} \hat{t}} d \hat{f}
$$

where $u(\boldsymbol{\rho}, \hat{f})$ is the solution of Eq. (1) at frequency $\hat{f}$ and $\hat{t} \equiv t c / a$ is the dimensionless time. The total energy stored in a particular cylinder can be evaluated from

$$
U(\hat{t})=\frac{\epsilon_{d}}{2} \int_{\text {cylinder }}|\tilde{u}(\boldsymbol{\rho}, \hat{t})|^{2} d \boldsymbol{\rho} .
$$

By using Eq. (12) we are able to study the resonant energy transfer between two defect cylinders for the three cases discussed in Fig. 2, i.e., resonance (i) between two defect states inside the gap $\left(\epsilon_{d}=6\right)$, (ii) between a defect state and a resonant state at the band edge $\left(\epsilon_{d}=5\right)$, and (iii) between the defect state and a resonant state inside the band $\left(\epsilon_{d}=4.4\right)$.

For case (i), we apply a Gaussian pulse with frequency centered at the middle between two defect frequencies $\hat{f}$ $=0.459$ and 0.513 with a width equal to half of their separation, i.e., $\hat{f}=0.486$ and $\hat{\delta}=0.027$. Using Eq. (12), we calculated the time dependence of the wave energies $U_{1}(\hat{t})$ and $U_{2}(\hat{t})$ stored in each of the two defect cylinders, where the subscript " 1 ", denotes the defect cylinder at the center of the sample. The results of $U_{1}(\hat{t})$ and $U_{2}(\hat{t})$ are plotted by solid and dashed curves, respectively, in the inset of Fig. 4(a). The oscillatory energy transfer between the two defect cylinders is clearly seen. The period of energy exchange is found to be $\Delta \hat{t} \approx 18.5$, which is precisely the inverse of the separation between two defect frequencies $\Delta \hat{f} \approx 0.054$. The total energy of the system remains nearly constant, as it has to be since the EM wave is injected in a frequency range forbidden in the photonic crystal. Apart from the oscillatory part, there also exists a time-independent nonoscillatory contribution to the energy stored in each of the defect cylinders. Its magnetide is close to 0.03 in the scale shown in the inset of Fig. 4(a). The existence of this nonoscillatory part can be understood by simplifying the problem as a two-level system. If we denote $\left|d_{l}\right\rangle$ and $\left|d_{u}\right\rangle$ as the defect states at frequencies $\hat{f}_{l}=0.459$ and $\hat{f}_{u}=0.513$, respectively, we can naively write the injected wave as $|\Psi\rangle \approx a\left|d_{l}\right\rangle+b\left|d_{u}\right\rangle$, where $a$ and $b$ are two real constants. In the case of $a=b, U_{1,2}(t)$ contains only oscillatory part and there will be complete energy transfer. Since the injected wave from the line source does not excited a single defect mode alone, therefore, we would expect $a$ $\neq b$ in general. In this case, the energy transfer becomes incomplete and there exists a finite energy minimum in $U_{1,2}(t)$ with a magnitude proportional to $(a-b)^{2}$.

Similar results are obtained for case (ii) and are plotted in Fig. 4(a). In this case, the oscillatory energy transfer has a period $\Delta \hat{t} \approx 17.2$, which is equal to the inverse of the separation between the defect state $\left|d_{l}\right\rangle$ inside the gap and the resonant state $\left|d_{u}\right\rangle$ at the band edge. Since the state $\left|d_{u}\right\rangle$ includes some bulk states, it can carry the energy away. Thus, the oscillatory term in each defect cylinder will decay to zero exponentially. In fact, the upper envelop can be well 

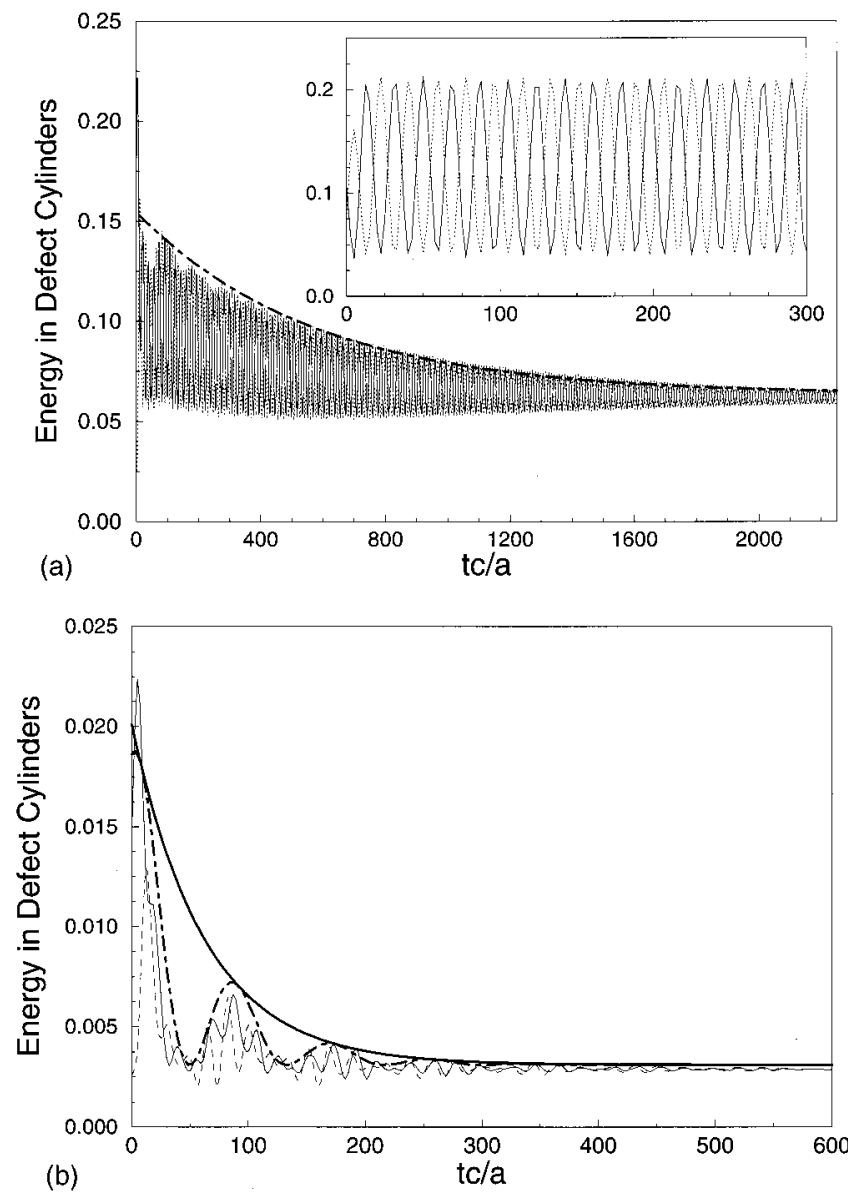

FIG. 4. After the injection of a Gaussian pulse at the origin, the time dependence of the energy stored in two defect cylinders for the cases (a) $\epsilon_{d}=5.0$, (b) $\epsilon_{d}=4.4$. The inset in (a) represents the case of $\epsilon_{d}=6.0$. The solid curves represent the defect cylinder at the origin and the dashed curves represent the other defect cylinder. The heavy dot-dashed curves in (a) and (b) are the fitted curved described in the text.

fitted by an exponential function of the form $0.09 e^{-0.0018 t}$ +0.06 as indicated by a heavy dot-dashed curve. From the fitting, we found a decay time $\hat{t}_{d} \approx 555$ units. The nonoscillatory part, which has the value $\approx 0.06$, will approach $a^{2}$ as the system evolves towards $\left|d_{l}\right\rangle$. This nonoscillatory part can also decay if the state $\left|d_{l}\right\rangle$ becomes leaky. This can occur if the sample size is small enough. In order to check this, we have repeated the calculations with the reduced sample radius from $R_{s}=6 a$ to $4 a$ and $3 a$. Our results have shown that the oscillatory energy transfer always exists in the early time. However, their upper and lower envelopes gradually merge to a single nonoscillatory curve which decays to zero in the long-time limit. As expected, the decay rate is faster as $R_{s}$ becomes smaller and system is leakier. It is also interesting to point out that the decay of oscillatory part is enhanced when the localized state $\left|d_{l}\right\rangle$ itself becomes leaky.

The results for case (iii) are plotted in Fig. 4(b). In this case, the frequency of the pulse spectrum is centered at the average of the defect state $\hat{f}_{c} \approx 0.498$ and the resonant state $\hat{f}_{c} \approx 0.556$. From Fig. 4(b) we find frequency modulation in addition to the energy leakage. The energy leakage occurs in a faster rate due to the presence of band-edge states at $\hat{f}_{c}$ $\approx 0.544$ in the vicinity of the resonant state. This band-edge state also introduces modulations in the energy transfer. The envelop of $U_{1}(t)$ can be fitted by the function $0.017 e^{-0.016 \hat{t}} \cos ^{2}(0.038 \hat{t}-0.3)+0.003$ as shown by a heavy dot-dashed curve in Fig. 4(b). Due to the existence of propagating band states at the edge, the decay time $\hat{t}_{d} \approx 62$ is much smaller than that shown in Fig. 4(a). So is the nonoscillatory energy stored in each cylinder $U_{1,2}(\infty)=0.003$. From the period of modulation $\Delta \hat{t} \approx 165$, we find the frequency of the modulation as $\Delta \hat{f}=2 / \Delta \hat{t} \approx 0.012$, which is equal to the separation between the band-edge state and the resonant state.

\section{SUMMARY}

In a photonic-band-gap system with two interacting defects, the incident EM waves have frequencies in the spectral gap, and are not allowable in the system except in the vicinity of the defects. The oscillatory energy transfer between the two defects may be thus viewed as tunneling through a barrier. The injection of an EM wave into one of the defects, and the subsequent oscillatory tunneling of the EM wave into the other defect, is a manifestation of the time evolution of nonstationary states. It is a classical analog of the time development of wave packets in a double-well potential found in many quantum texts. The observation of all these resonant behaviors can, in principle, be realized in fairly simple 2D microwave experimental setups, and can provide a vivid demonstration of the phenomenon that has bewildered many beginning students in quantum mechanics, while highlighting the underlying similarity between classical wave and quantum physics. This case is actually richer in phenomena, since the possibility of frequency modulation and energy leakage can be incorporated naturally when one defect state merges with the pass band continuum.

In experimental situations, the cylinders are finite in length. This will introduce some mixing of the TE mode and provide an additional loss mechanism. However, if the length of the cylinders is an order of magnitude larger than the wavelength of interest here, the effects due to mixing would be small and will not alter the main features presented here. The mixing may be avoided if we switch to a structure that support an absolute gap for both TM and TE modes such as the honeycomb structure. ${ }^{10}$ In this case the defect modes for TM and TE appear at different frequencies and the mixing of modes becomes negligible.

\section{ACKNOWLEDGMENTS}

Z.Q.Z. and C.T.C. acknowledge the support of this work by RGC Grants Nos. HKUST6137/97P and HKUST6136/ 97P, respectively. 
*Permanent address: Department of Modern Applied Physics, Tsinghua University, Beijing 100084, China.

${ }^{1}$ For example, C. Cohen-Tannoudji, B. Diu, and F. LaLoe, Quantum Mechanics (Wiley, New York, 1997).

${ }^{2}$ For example, W. A. Philips, Rep. Prog. Phys. 50, 1657 (1987).

${ }^{3}$ Photonic Band Gap Materials, edited by C. M. Soukoulis (Kluwer Academic, Dordrecht, 1996).

${ }^{4}$ E. Yablonovitch, Phys. Rev. Lett. 58, 2059 (1987).

${ }^{5}$ S. John, Phys. Rev. Lett. 58, 2486 (1987).

${ }^{6}$ K. M. Ho, C. T. Chan, and C. M. Soukoulis, Phys. Rev. Lett. 65, 3152 (1990).

${ }^{7}$ W. M. Robertson, G. Arjavalingam, R. D. Meade, K. D. Brommer, A. M. Rappe, and J. D. Joannopoulos, Phys. Rev. Lett. 68, 2023 (1992).

${ }^{8}$ S. L. McCall, P. M. Platzman, R. Dalichaouch, D. Smith, and S. Schultz, Phys. Rev. Lett. 67, 2017 (1991); J. Mod. Opt. 41, 395 (1994); also S. Schultz and D. R. Smith, in Photonic Band Gaps and Localization, edited by C. M. Soukoulis (Plenum, New York, 1993).

${ }^{9}$ M. Sigalas, C. M. Soukoulis, E. M. Economou, C. T. Chan, and K. M. Ho, Phys. Rev. B 48, 14121 (1993).

${ }^{10}$ G. Taylb and D. Maystre, J. Opt. Soc. Am. A 14, 3323 (1997).

${ }^{11}$ Y. S. Chan, C. T. Chan, and Z. Y. Liu, Phys. Rev. Lett. 80, 956 (1998).

${ }^{12}$ J. D. Joannopoulos, R. D. Meade, and J. N. Winn, Photonic Crystals: Molding the Flow of Light (Princeton University Press, Princeton, 1995).

${ }^{13}$ L. M. Li and Z. Q. Zhang, Phys. Rev. B 58, 9587 (1998).

${ }^{14}$ G. Kurizki and A. Z. Genack, Phys. Rev. Lett. 61, 2269 (1988).

${ }^{15}$ S. John and J. Wang, Phys. Rev. Lett. 64, 2418 (1990).

${ }^{16}$ S. John and T. Quang, Phys. Rev. Lett. 74, 3419 (1995); 76, 1320 (1996).

${ }^{17}$ S. Y. Zhu, H. Chen, and H. Hu, Phys. Rev. Lett. , 205 (1997). 